\title{
Extraction of Quantitative Parameters for Describing the Microstructure of Solid Oxide Fuel Cells
}

\author{
Seung-Muk Bae, Yong-Hoon Kim, Yil-Hwan You, and Jin-Ha Hwang* \\ Department of Materials Science and Engineering, Hongik University 72-1 Sangsu-Dong, Mapo-Gu, Seoul 121-791, Korea
}

\begin{abstract}
Digital quantification of a two-dimensional structure was applied to a $\mathrm{GDC}\left(\mathrm{Gd}_{2} \mathrm{O}_{3}\right.$-doped $\left.\mathrm{CeO}_{2}\right)$ / $\operatorname{LSM}\left(\mathrm{La}_{0.85} \mathrm{Sr}_{0.15} \mathrm{MnO}_{3}\right)$ composite cathode employed for solid oxide fuel cells. With the aid of high-resolution imaging capability based on secondary and backscattered electron images, two-dimensional electron micrographs were converted to digital binary files using an image processing tool combined with the line intercept method. Statistical analysis combined with a metallurgical tool was employed to determine microstructural factors, i.e., volume fraction, size distribution, and interconnectivity. The current work reports the quantification of the two-dimensional structural images of GDC/LSM composites applicable to solid oxide fuel cells, with the aim of obtaining the volume fraction, size distribution, and interconnectivity as functions of composite composition. The volume fractions of the solid constituent phases exhibit compositional dependence in cathodes; however, LSM interconnectivity increases gradually as a function of LSM composition, whereas that of GDC decreases significantly at $50 \mathrm{wt} \%$ LSM.
\end{abstract}

Key words: image processing, digital processing, microstructural factors, solid oxide fuel cells, cathodes, line intercepts

\section{INTRODUCTION}

An energy crisis in the 21st-century economy has closely been related to the ever-increasing rise in petroleum prices and relentless pollution. Practical solutions have been attempted in the form of renewable energy to resolve the current economic and ecological issues. Renewable energy sources have introduced a green energy term, "hydrogen energy." Renewable energy has been attracting extensive interest from academia and industry; particularly, functional electroceramics have possible applications in solid oxide fuel cells (SOFCs), water-splitting materials, and oxygen transport membranes (Brown, 1986; Naito \& Arashi, 1995; Berman \& Epstein, 2000; Shao et al., 2000; Steele \& Heinzel, 2001; Abanades \& Flamant, 2006; Balachandran et al., 2007). However, the operating principles of the abovementioned applications rely critically on the point defect chemistry of the solid electrolytes, electrodes, and membranes. The SOFCs are constructed using ionic, electronic conductors, and mixed conductors, including even metallic components. Unlike dense electrolytes, the electrodes, i.e., the cathodes and anodes of SOFCs, are multiphase systems that typically comprise three components: an electrolyte, an electronic (or mixed) conductor, and a porous phase. The optimization of SOFC electrodes relies on the size, distribution, and amount of the constituent phases. The overall microstructure affects the electrochemical performance, which influences output performance and long-term stability at high temperatures. Because pores function as major pathways for reactants and products, gas-related phenomena (i.e., gas permeability, diffusivity, and permeability) are critically dependent on the size and distribution of porous

(c) MICROSCOPY SOCIETY OF AMERICA 2013

${ }^{\star}$ Corresponding author. E-mail: jhwang@wow.hongik.ac.kr phases. The significant issues in electrocomposites can be found in Newnham's review (McLachlan et al., 1990).

This study placed a special focus on the quantitative characterization of a multiphase cathode material system made up of an electrolyte and an electronic conductor, i.e., $\mathrm{Gd}_{2} \mathrm{O}_{3}$ doped $\mathrm{CeO}_{2}\left(\mathrm{GDC}: \mathrm{Ce}_{0.9} \mathrm{Gd}_{0.1} \mathrm{O}_{1.95}\right.$ ) and $\mathrm{La}_{0.85} \mathrm{Sr}_{0.15} \mathrm{MnO}_{3}$ (LSM), in addition to an inherent porous component. The GDC/LSM mixtures can be applied to electrodes in intermediate-temperature SOFCs. The electrochemical behavior of the GDC/LSM is affected by the corresponding microstructure in addition to the relative amounts of GDC and the electronic conductor, LSM. Accordingly, this study places a special emphasis on the microstructural characterization of GDC/LSM through statistical/digitized image analyses. Quantified image analysis allows for the provision of quantitative explanations for the size, volume fraction, and interconnectivity of the constituent phases. The numerical image analysis parameters can be applied to a variety of electrode composites with the aim of optimizing the electrochemical performance, i.e., the output characteristics, and to enhance their corresponding long-term stability under harsh conditions. An exemplary work of electrode materials is analyzed systematically and explicitly with regard to a systematic description of the multiphase materials.

\section{EXPERIMENTAL}

In order to prepare the GDC/LSM cathode composites, mixtures were prepared using $\mathrm{Gd}_{2} \mathrm{O}_{3}$-doped $\mathrm{CeO}_{2}$ (Rhodia, France) and $\mathrm{La}_{0.85} \mathrm{Sr}_{0.15} \mathrm{MnO}_{3}$. LSM composition was controlled as a function of the weight percent, i.e., 30, 50, and $70 \mathrm{wt} \%$, with regard to the remaining GDC component. The powder mixtures for each composition were subjected to a ball-milling procedure with a dispersing agent includ- 

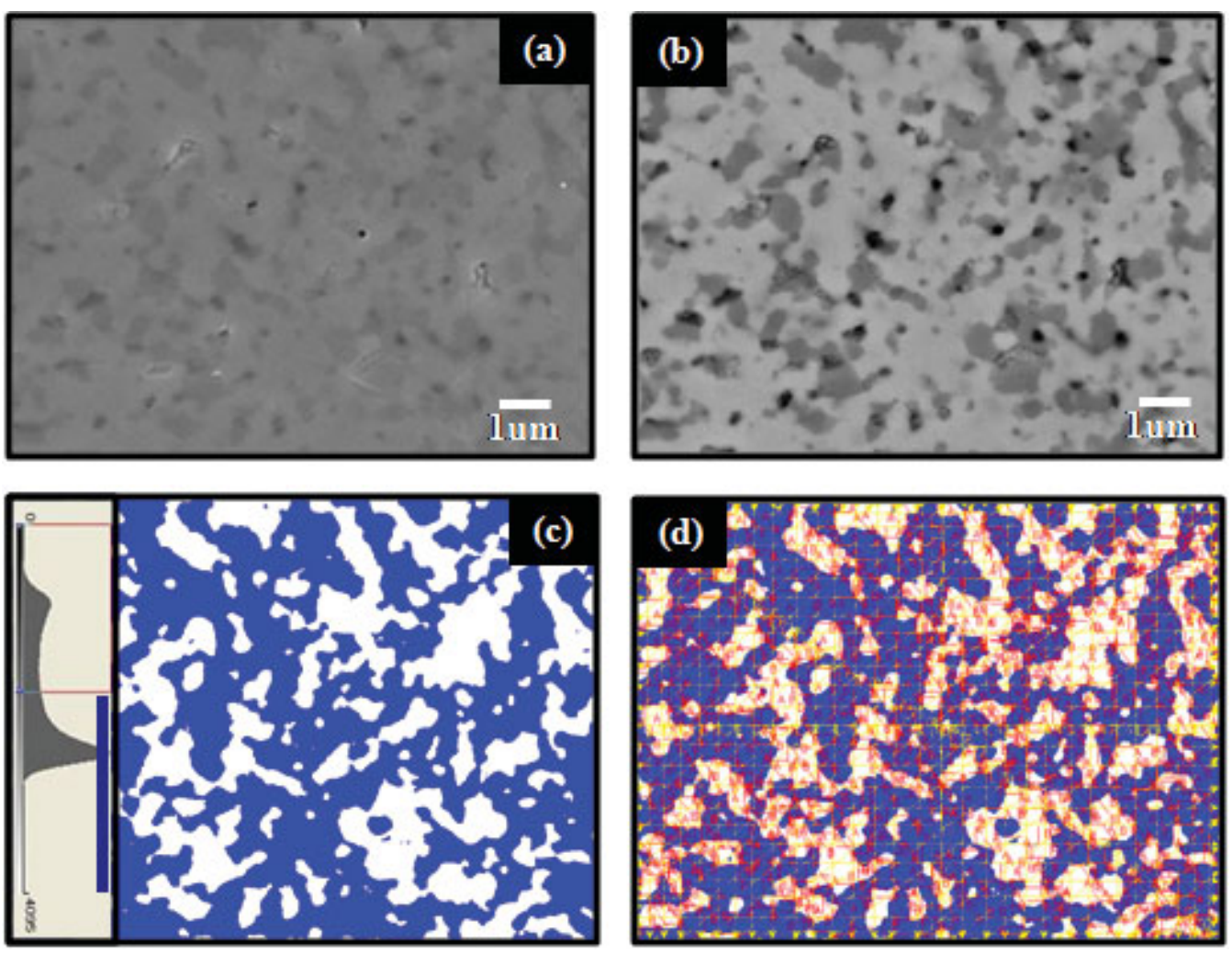

Figure 1. Scanning electron images captured in two different modes on solid oxide fuel cells cathode [GDC (70 wt $\%)$ / LSM (30 wt \%) composite]: (a) secondary electron image, (b) backscattered electron image, (c) binary image after noise filtering (grayscale images are optimized into binary ones through high contrasts between the constituent phases), and (d) linear line intercepts for statistical GDC phase image analysis in GDC (70 wt\%)/LSM (30 wt\%) component.

ing appropriate wear-resistant zirconia balls. The ballmilled mixtures were dried at a temperature of $105^{\circ} \mathrm{C}$ in order to eliminate the dispersing materials. The dried powder mixtures were pressed into $10-\mathrm{mm}$ disc-shaped pellets at a pressure of $5 \mathrm{MPa}$. The pressed specimens were heated to a temperature of $1,150^{\circ} \mathrm{C}$ in ambient conditions for $10 \mathrm{~h}$.

After heat treatment, the porous space of the sintered GDC/LSM composite specimens was infiltrated with an epoxy resin in order to provide appropriate rigidity against subsequent polishing. The molded specimens were polished to $0.25 \mathrm{~m}$ using $\mathrm{SiC}$ abrasive papers and diamond pastes to obtain highly polished surfaces. The polished cathode surfaces were then monitored by high-resolution fieldemission scanning electron microscopy (JEOL, JSM-6700F, Tokyo, Japan). The digital images were acquired in two complementary modes: secondary electron images and backscattered electron images. The typical acceleration voltage was fixed at $15 \mathrm{keV}$ to maximize the contrast between the constituent phases, i.e., pores, GDC, and LSM. Commercially available image software (Image-Pro, Media Cybernetics, USA) was employed to perform refined image processing and quantitative analysis in order to statistically extract the numerical parameters based on the two-dimensional images; the software was applied to image capturing, image optimization, and data acquisition analysis in digital processing.

\section{Results And Discussion}

Traditionally, scanning electron microscopy has been widely employed in materials science and engineering, mainly for qualitative analyses, owing to its analytically visual capability and its relative ease of operation. Scanning electron microscopy can be operated either in secondary or baskscattered electron modes. The lateral resolution keeps increasing along with advanced developments in electronics and novel concepts in sensing components, i.e., field-emission guns as electron-generating sources and electron backscattered electron diffraction techniques as new approaches for quantifying microstructural orientations (Brundle et al., 1992; Goldstein et al., 2003). In particular, the enhanced electron emissions in field-emission sources can be operated in conjunction with backscattered and secondary electron modes.

The typical secondary and backscattered electron images of an SOFC cathode composite are shown in Figures 1a and $1 \mathrm{~b}$. Owing to the origins in the corresponding modes, the backscattered electron image is of a higher resolution and contrast; the backscattered electrons are highly sensitive to atomic number unlike the secondary electrons, which are sensitive to the surface features of the materials under investigation. Such enhanced features in backscattered electrons can be adapted to image processing (or to resolve the 
corresponding component phases) in order to proceed with the statistical analyses based on two-dimensional information. The quantitative analyses are composed of two parts, i.e., image processing for optimized image states and quantification steps for microstructural parameters. The first image processing step is divided into image capturing, scale calibration, and digitized image processing. The image capturing and scale calibration is relatively straightforward upon adopting the microstructural image based on the backscattered electron mode. The high-contrast digitized image processing depends on the discreet separation of constituent phases, in this work the pores, the electrolyte (GDC), and the electrode (LSM). Within this stage, the "thresholding" step is extremely critical because unbalanced images result in overestimation of one component and underestimation of the remaining components. Figure 1c shows detailed information pertaining to the "thresholding" steps. In "thresholding" procedure, binary images were monitored by adjusting the boundary condition in grayscale images between two adjacent phases; the contrast was set to high in order to accurately reflect the original microstructural information. After strict comparison with the original images, the images are converted into binary modes where the existence of the corresponding phase is denoted by " 1 " and the remaining counterpart denoted by " 0 ."

An acquired image can be deconvoluted into the three constituents: pores, LSM phase, and GDC phase: each phase component image is compared with the original image micrograph in order to enhance the accuracy in the conversion to binary images. The optimized binary images were subjected to a statistical analysis stage in which the primary experimental input variables are obtained, i.e., surface fraction and line intercepts. The corresponding surface fractions are added in a cumulative manner and normalized with regard to the given two-dimensional areas (not shown here). Another parameter, a mean line intercept, is calculated after mutual interconnection of the random lines with the corresponding phases in which the lines are only meaningful when they are located within the given phases. In order to collect statistically significant data values, the lines are placed onto the binary images, at least 91 lines in the horizontal, perpendicular, and diagonal directions (see Fig. 1d). The obtained parameters can be combined in order to estimate the interconnectivity of the constituent phases in which the three-dimensional contiguity is quantified between 0 and 1 . The value of 0 denotes the isolation of the constituent phases and the value of 1 denotes the straight linear tunneling across two endpoints. The calculated area fractions and mean line intercepts are given in three GDC/ LSM components. Furthermore, the current microstructural analysis is based on the isotropic features in which the shapes and distribution are isotropic in the three components.

The statistical data were combined with conventional metallurgy concepts in order to provide phase fraction, size distribution, and interconnectivity (Smith \& Guttman, 1953; Cahn \& Hilliard, 1959; Alexander et al., 1994; Simwonis et al., 2000). Those three parameters determine the overall physical properties, e.g., electrical conductivity, dielectric constant, mechanical strength, etc. Owing to the isotropic property of the constituent phases, the surface area can be assumed to be identical to the three-dimensional volume fractions. Therefore, the volume fraction and mean intercepts are determined experimentally, leading to the estimation of the fraction of the contact area, $S_{V}^{i}$, according to the following equation:

$$
\frac{V_{i}}{S_{V}^{i}}=\frac{l_{i}}{4}
$$

Thus, the experimentally determined line intercepts are employed in order to calculate the three-dimensional interconnection of the core components. The basic interconnectivity is defined by

$$
\beta_{i}=\frac{S_{V}^{i}}{\sum_{i} S_{V}^{i}} .
$$

According to the above relationships and the concept of our previous work (Lee et al., 2005), the corresponding interconnectivities of the GDC/LSM cathode systems are given in the following equations:

$$
\begin{gathered}
\beta_{\mathrm{GDC}}=\frac{V_{\mathrm{GDC}} l_{\mathrm{LSM}} l_{\text {Pore }}}{V_{\mathrm{LSM}} l_{\mathrm{GDC}} l_{\text {Pore }}+V_{\mathrm{GDC}} l_{\mathrm{LSM}} l_{\text {Pore }}+V_{\text {Pore }} l_{\mathrm{LSM}} l_{\mathrm{GDC}}}, \\
\beta_{\mathrm{LSM}}=\frac{V_{\mathrm{LSM}} l_{\mathrm{GDC}} l_{\text {Pore }}}{V_{\mathrm{LSM}} l_{\mathrm{GDC}} l_{\text {Pore }}+V_{\mathrm{GDC}} l_{\mathrm{LSM}} l_{\text {Pore }}+V_{\text {Pore }} l_{\mathrm{LSM}} l_{\mathrm{GDC}}}, \\
\beta_{\text {Pore }}=\frac{V_{\text {Pore }} l_{\mathrm{GDC}} l_{\mathrm{LSM}}}{V_{\mathrm{LSM}} l_{\mathrm{GDC}} l_{\text {Pore }}+V_{\mathrm{GDC}} l_{\mathrm{LSM}} l_{\text {Pore }}+V_{\text {Pore }} l_{\mathrm{LSM}} l_{\mathrm{GDC}}} .
\end{gathered}
$$

Three different cathode composites are chosen in order to test the current approaches, i.e., GDC(70 wt $\%) / \operatorname{LSM}(30$ wt $\%), \operatorname{GDC}(50 \mathrm{wt} \%) / \operatorname{LSM}(50 \mathrm{wt} \%)$, and GDC(30 wt $\%) /$ $\operatorname{LSM}(70 \mathrm{wt} \%)$. The original BSE images and digitized images are shown in Figure 2. The resultant information is summarized in Figure 3. As the current cathode composites are not optimized as cathodes, the volume fraction and interconnectivity of the pore components are relatively small. However, the volume fractions and interconnectivities of the GDC and LSM components exhibit an overall change depending on the change in composition. The GDC interconnectivity decreases from 0.619 to 0.135 upon changing from 70 to $30 \mathrm{wt} \%$, and simultaneously the corresponding surface fractions decrease from 0.599 to 0.235 .

The GDC/LSM composites were chosen as the cathode model system because the two different electrical features can contribute to the significant role of the SOFC unit cells. Ultimately, the current work was performed using microscale images obtained using scanning electron microscopy. The facilitated image analysis technique can be beneficial in 

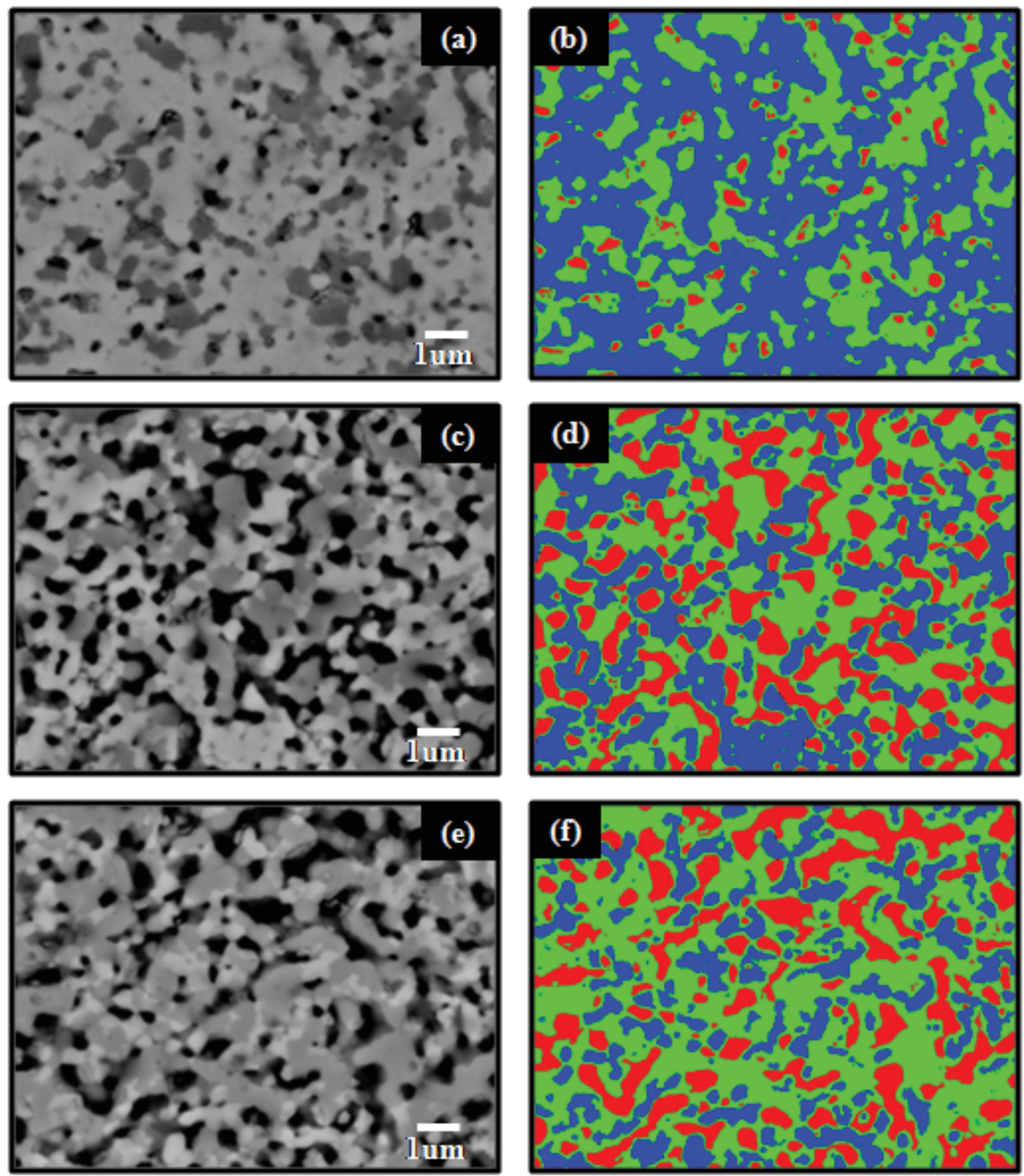

Figure 2. (a), (c), (e) High-contrast backscattered electron images and (b), (d), (f) digitized images for image analysis in three GDC/LSM cathode composites: (a) and (b) for GDC (70 wt\%)/LSM (30 wt \%); (c) and (d) for GDC (50 wt $\%)$ / LSM (50 wt $\%$ ); and (e) and (f) for GDC (30 wt \%)/LSM (70 wt \%). For the constituent phase components: pores (red color), LSM phase (green color), and GDC components (blue color).

optimizing the output performance of an SOFC cell. The quantitative characterization and artificial control in microstructure will be exploited in a sophisticated manner.

In summary, a two-dimensional image analysis can be performed based on the area fractions and the linear intercepts, leading to the three-dimensional microstructural quantifications in multiphase systems, i.e., the relative fraction, size distribution, and interconnectivity (or contiguity). Furthermore, the quantitative microstructural control in SOFC electrodes, i.e., both cathodes and/or anodes, is closely related to the gas transport optimization of electrodes in terms of the delivery of reactants and the removal of reactants in SOFCs and the balanced electrochemical reactions through the appropriate control of ionic and electronic conductions in producing oxygen ions under the operating conditions in SOFCs.

\section{CONCLUSIONS}

Image processing in conjunction with scanning electron microscopy was attempted with the aim of quantifying the microstructural properties of energy-oriented composites, i.e., SOFC cathodes and GDC/LSM cathode composite mixtures. Because of the relatively poor contrast in secondary images, high-contrast backscattered images were employed in digitizing the microstructural images involving two solid phases and a porous component. The multiphase cathode composites were quantitatively analyzed in terms of the 


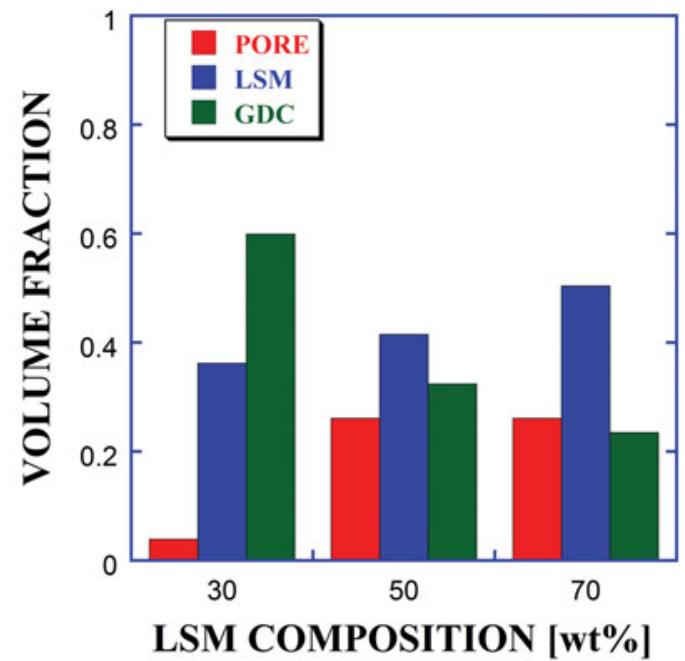

(a)

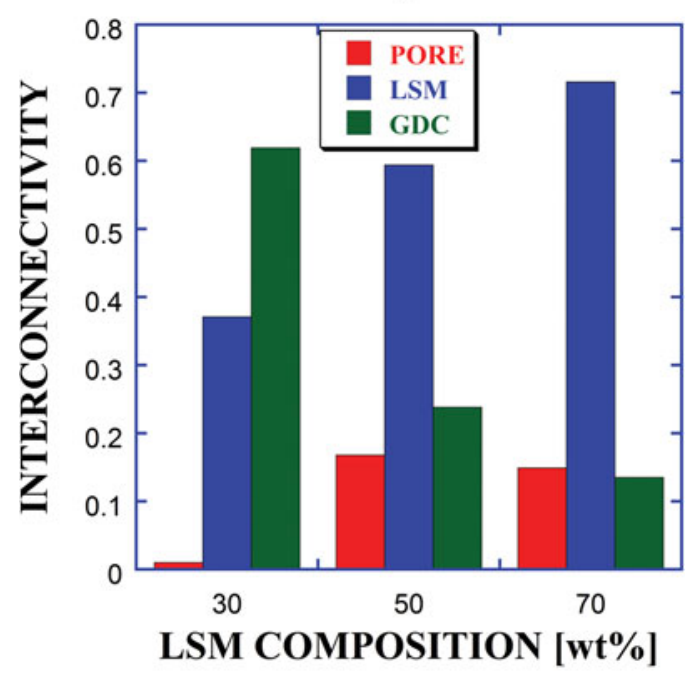

(b)

Figure 3. Calculated microstructural parameters: (a) volume fraction and (b) interconnectivity as a function of cathode composition.

volume fraction, size, and distribution, along with the interconnectivity of the corresponding constituents, i.e., GDC, LSM, and pore. The volume fractions of the solid constituent phases exhibit the compositional dependence in cathodes; however, LSM interconnectivity increases gradually as a function of LSM composition, whereas that of GDC decreases significantly at $50 \mathrm{wt} \%$ LSM.

\section{ACKNOWLEDGMENTS}

This research was supported by a grant from the Fundamental R\&D Program for Core Technology of Materials funded by the Ministry of Knowledge Economy and by the Converging Research Center Program through the Ministry of Education, Science and Technology (2012K001296).

\section{REFERENCES}

Abanades, S. \& Flamant, G. (2006). Thermochemical hydrogen production from a two-step solar-driven water-splitting cycle based on cerium oxides. Solar Energy 80, 1611-1623.

Alexander, K.B., Becher, P.F., Waters, S.B. \& Bleier, A. (1994). Grain growth kinetics in alumina-zirconia (CeZTA) composites. J Am Ceram Soc 77, 939-946.

Balachandran, U., Lee, T.H. \& Dorris, S.E. (2007). Hydrogen production by water dissociation using mixed conducting dense ceramic membranes. Int J Hydrogen Energy 32, 451-456.

Berman, A. \& Epstein, M. (2000). The kinetics of hydrogen production in the oxidation of liquid zinc with water vapor. Int J Hydrogen Energy 25, 957-967.

Brown, J.T. (1986). High-temperature solid-oxide fuel cell. Energy 11, 209-229.

Brundle, C.R., Evans, C.A., JR. \& Wilson, S. (1992). Encyclopedia of Materials Characterization. Greenwich, CT: Manning Publications Co.

Cahn, J.W. \& Hilliard, J.E. (1959). The measurement of grain contiguity in opaque samples. Trans Metall Soc AIME 215, 759-765.

Goldstein, J., Newbury, D., Joy, D., Lyman, C., Echlin, P., Lifshin, E., Sawyer, L. \& Michael, J. (2003). Scanning Electron Microscopy and X-Ray Microanalysis. New York: Kluwer Academic/Plenum Publishers.

Lee, K.-R., Choi, S.H., Kim, J., Lee, H.W. \& Lee, J.-H. (2005). Viable image analyzing method to characterize the microstructure and the properties of the Ni/YSZ cermet anode of SOFC. J Power Sources 140, 226-234.

McLachlan, D.S., Blaszkiewicz, M. \& Newnham, R.E. (1990). Electrical resistivity of composites. J Am Ceram Soc 73(8), 2187-2203.

Naito, H. \& Arashi, H. (1995). Hydrogen production from direct water splitting at high temperatures using a $\mathrm{ZrO}_{2}-\mathrm{TiO}_{2}-\mathrm{Y}_{2} \mathrm{O}_{3}$ membrane. Solid State Ionics 79, 366-370.

Shao, Z., Yang, W., Cong, Y., Dong, H., Tong, J. \& Xiong, G. (2000). Investigation of the permeation behavior and stability of a $\mathrm{Ba}_{0.5} \mathrm{Sr}_{0.5} \mathrm{Co}_{0.8} \mathrm{Fe}_{0.2} \mathrm{O}_{3-\delta}$ oxygen membrane. $J$ Membrane Sci 172(1-2), 177-188.

Simwonis, D., Tietz, F. \& Tagawa, H. (2000). Nickel coarsening in annealed Ni/8YSZ anode substrates for solid oxide fuel cell. Solid State Ionics 132, 241-251.

Smith, C.S. \& Guttman, L. (1953). Measurement of internal boundaries in three-dimensional structures by random sectioning. Trans Metall Soc AIME 197, 81-87.

Steele, B.C.H. \& Heinzel, A. (2001). Materials for fuel-cell technologies. Nature 414, 345-352. 